\title{
Los cuerpos cíborgs en cuatro cuentos de ciencia ficción costarricense
}

Por Diana Martínez Alpízar', Universidad de Costa Rica

Recibido: 15 de enero, 2018.

Aceptado: 25 de marzo, 2018.

\section{Resumen}

Género poco estudiado en el ámbito nacional, la ciencia ficción en Costa Rica es aún un campo por explotar. Justamente, en este artículo se analizan cuatro cuentos de dicho género, publicados a inicios del siglo XXI: "Yo, la criatura" de José Ricardo Chaves; "Joyería mental" de Jessica Clark; "Lunas en vez de sombras" de Ericka Lippi y "El ejército de Onara" de Daniel Garro. Todos ellos tienen algo en común: la presencia de cuerpos cíborgs, objeto de interés en el presente análisis. Para este propósito, en un primer lugar se teoriza sucintamente sobre el género ciencia ficción; posteriormente, se explica el concepto de cuerpos cíborgs y su relación con lo poshumano, con base en los aportes teóricos de Donna Haraway, Rosi Braidotti, Katherine Hayles y Christian Hables Gray, entre otros. En cuanto al análisis literario del corpus, se examina la construcción y la descripción, a través de distintos recursos estilísticos, de los cuerpos de los personajes en los distintos textos. Se concluye que el corpus analizado, a partir de la lectura propuesta, muestra una serie de reflexiones sobre las tensiones, miedos, preocupaciones y posibilidades sobre el desarrollo científico y tecnológico asociado a las intervenciones y modificaciones en el cuerpo humano.

\section{Abstract \\ The Cyborg Bodies in four Costa Rican science fiction short histories}

Largely unexplored in the domestic field, the field of science fiction in Costa Rican Literature offers many opportunities for research. This article analyzes four Costa Rican science fiction short stories published in the early 21 st century: "Yo, la criatura" by José Ricardo Chaves, "Joyería mental" by Jessica Clark; "Lunas en vez de sombras" by Ericka Lippi, and "El ejército de Onara" by Daniel Garro. All of these works have something in common: the presence of cyborgs, the focus of this analysis. To this effect, firstly it offers a brief theorization about science fiction; hereinafter, the notion of cyborg and its relation with post-humanity are explained, based on authors Donna Haraway, Rosi Braidotti, Katherine Hayles, and Christian Hables Gray, among others. Regarding literary analysis, this article focuses on construction and description, through the stylistic resources of the bodies of the characters in the short stories, offering an individual interpretation of each one and closing with a comparison between the different propositions. It concludes that these short stories show different reflections, tensions, fears, concerns and possibilities on science and technology linked with the modification and intervention of the human body.

1 Bachiller en Filología Española y Máster en Literatura Latinoamericana, ambos títulos obtenidos en la Universidad de Costa Rica. Actualmente labora en esta misma institución, en la Escuela de Estudios Generales. Contacto: dimartinezalpizar@gmail.com.
Diana Martínez Alpízar. Los cuerpos cíborgs en cuatro cuentos de ciencia ficción costarricense. Revista Comunicación. Año 39, volumen 27, número 1, enero a junio, 2018. Instituto Tecnológico de Costa Rica. ISSN: 0379-3974 / e-ISSN1659-3820.
PALABRAS CLAVE:

Ciencia ficción, cuento, análisis literario, cibernética, literatura latinoamericana

\section{KEY WORDS:}

Science fiction, short stories, literary analysis, cybernetics, Latin American literature. 


\section{INTRODUCCIÓN}

Las tensiones y relaciones entre literatura, ciencia y desarrollo tecnológico no son ninguna novedad en el contexto latinoamericano. Basta recordar la aparición, en 1879, de la novela Horacio Kalibang o los autómatas, escrita por el argentino Eduardo Holmberg, texto que -según Brown (2010) - inaugura la ciencia ficción en la literatura hispanoamericana.

En el caso de la literatura nacional, según Molina (2010 y 2015), es posible rastrear los orígenes de la ciencia ficción en la literatura costarricense desde finales del siglo XIX e inicios del siglo XX con textos como el cuento "El número 13,013" (1908) de León Fernández Guardia o bien, las novelas El problema, del guatemalteco Máximo Soto Hall, pero publicada en Costa Rica en 1899 y La caída del águila (1920) de Carlos Gagini. No obstante, hay que dejar que el tiempo transcurra, pues es en la década de 1990 cuando el género toma fuerza para, según Pacheco Solórzano (2014), renovarse discursivamente con los autores que publican a inicios del siglo XXI, cuyos textos muestran "un salto cualitativo" (p. 65) en relación con producciones anteriores.

En contraste con este auge del género a finales del siglo XX e inicios del XXI, la academia costarricense se ha acercado tímidamente a su estudio. Si bien especialistas como Víquez Jiménez (2014), Soto Bogantes (2014), Ruiz Ulloa (2014), Cuvardic García (2014), Alfaro Vargas (2014) y el mismo Molina (2010 y 2015) se han dedicado a analizar diversos textos de este género, la labor de profundizar en su análisis en la literatura costarricense está en ciernes.

Justamente, este artículo se interesa por indagar en un tema particular de la ciencia ficción costarricense: aquellos cuerpos ficcionales que, a través de la intervención tecno-científica, difuminan o ponen entredicho los límites entre la naturaleza y la tecnología: ¿son máquinas o humanos, o quizá un "algo" diferente? Este "algo diferente" refiere precisamente a la presencia de cuerpos cíborgs en un corpus particular de cuentos escritos por los autores José Ricardo Chaves, Jessica Clarke, Ericka Lippi y Daniel Garro. La muestra seleccionada es representativa y no exhaustiva, claro está. Queda para futuros artículos ahondar en ello.
Con el propósito de analizar dichos cuerpos, el recorrido teórico-metodológico será el siguiente: en un primer lugar, se referirá brevemente a la noción de "ciencia ficción", con la intención no de agotar el tema -misión ya de por sí imposible- sino más bien con el propósito de establecer delimitaciones conceptuales inherentes al artículo. Posteriormente, se enriquece la discusión teórica con la aproximación al concepto cíborg y su relación con lo poshumano, con base en autores como Haraway (1984), Hayles (1999), Gray (1999, 2011), Sádaba (2009) y Braidotti (2015), entre otros.

\section{BREVE TEORIZACIÓN BÁSICA}

Uno de los primeros puntos de análisis yace en la definición del término "ciencia ficción", categoría en la cual se incluirían todos los cuentos del corpus seleccionado. La polémica sobre este concepto es amplia, por lo que para fines pragmáticos se recurre a dos autores cuya visión del género es compatible. En primer lugar, Eco (1998) se refiere a la ciencia ficción como un género en el que, a partir de las tendencias del mundo actual, extrapola otras que podrían desarrollarse en el futuro. Este carácter conjetural es tanto la esencia del género en sí como del quehacer científico: "En otros términos, la ciencia ficción es una narrativa de la hipótesis, de la conjetura o de la abducción y en ese sentido es juego científico por excelencia, dado que toda ciencia funciona mediante conjeturas, esto es abducciones" (Eco, 1998, p. 654).

Moreno (2010), por su parte-Gunn (2005) y Malmgren (1988)- explica que la ciencia ficción se caracteriza principalmente por "la inclusión de elementos no existentes en nuestra realidad inmediata, pero considerados "posibles" desde algún ámbito del conocimiento científico" (p. 106). Al igual que Eco, Moreno (2010) da especial valor a la proyección basada en elementos no sobrenaturales para caracterizar al género. Además y otra vez en coincidencia con Eco, Moreno entiende "conocimiento científico" en un sentido extenso; es decir, no lo reduce a las ciencias duras, sino que lo aplica también a otras áreas (sociología, lingüística, historia, etc). También es importante destacar otra aclaración de Moreno: poco importa que el "descubrimiento tecnológico" del cual se hable quede desfasado; pues, 
a través de un pacto de lectura con el texto, es en el conflicto entre el "descubrimiento tecnológico" y los personajes donde yace la relevancia del género.

Dentro del mismo género de ciencia ficción, hay temas recurrentes. Uno de ellos es la creación de cuerpos cíborgs. ¿De dónde proviene este término, popularizado en el ámbito académico por Donna Haraway en los 80? El origen de la palabra se remonta en realidad a los años 60 , en el contexto de la carrera espacial durante la Guerra Fría. Como explican Gray (1999) y Sádaba (2009), el término fue acuñado originalmente por Manfred Clynes y Nathan Kline, científicos en ese entonces de la NASA, quienes en un artículo argumentaban que los cuerpos humanos podrían ser modificados -a través de implantes y drogas- con el fin de que pudieran vivir en el espacio exterior sin necesidad de trajes espaciales. De esta forma, ellos propusieron la creación de cíborgs, palabra compuesta de la contracción de cybernetic organisms, cuya traducción sería "organismos cibernéticos".

A grandes rasgos, con el fin de aclarar el concepto del inicio, si bien hay distintas aproximaciones al cíborg, todas coinciden en que este se constituye a través de la mediación de la tecnología en un cuerpo biológico. Aunque la palabra correcta, como precisa Sádaba (2009), no es mediación, sino integración:

En general, entenderemos por cyborg la integración hombre-máquina en sus diferentes vertientes y versiones, un tipo especial de relación (individual y social) entre los sistemas naturales (biológicos, orgánicos) y los sistemas inorgánicos o técnicos (mecánicos, eléctricos, digitales, etc.) en la cual se diluyen las fronteras de los mismos (p. 35).

Propiamente en el ámbito académico y político, el cíborg será retomado y reconceptualizado como una categoría de análisis, al mismo tiempo que como una metáfora de las rápidas y constantes transformaciones tecnológicas y científicas. En este desarrollo, es necesario mencionar en un primer lugar a la feminista estadounidense Donna Haraway y su ya famoso Manifiesto cíborg. En él, Haraway (1984) entiende el cíborg como "un organismo ci- bernético, un híbrido de máquina y organismo, una criatura de realidad social y también de ficción" (p. 2). Este ser quimérico y utópico, invisible, pero ubicuo (de ahí su carácter mortal, apunta Haraway) confunde no solo los límites fundantes de las dicotomías natural-artificial, sino todas aquellas divisiones binarias del pensamiento occidental:

(...) entre la mente y el cuerpo, lo animal y lo humano, el organismo y la máquina, lo público y lo privado, la naturaleza y la cultura, los hombres y las mujeres, lo primitivo y lo civilizado están puestas ideológicamente entredicho (...) (Haraway, 1984, p. 18).

Como bien explica Sádaba (2009), Haraway ve en el cíborg la posibilidad de crear nuevas subjetividades, distantes tanto de las divisiones dicotómicas y jerarquizadas de la modernidad como del sujeto cartesiano, racional. Con propuestas muy vinculadas a la de Haraway, Katherine Hayles (1999) y Rosi Braidotti (2015) también se interesan por los cuerpos cíborgs, pero utilizan el término poshumano para referirse no tanto a un cuerpo modificado, sino a una etapa de la evolución humana, caracterizada por la imbricación entre los cuerpos biológicos y las máquinas, que además contempla una interacción con el entorno, todo parte de un mismo sistema. En lo poshumano, según Mejía (2005), al igual que con la propuesta cíborg de Haraway, se pone en jaque la idea del cuerpo humano propias del humanismo moderno.

Para entender esta idea de cuerpos poshumanos y por ende, cíborgs, se analiza brevemente las propuestas de Halyes (1999) y Braidotti (2015). Con coincidencias en diversos puntos, las dos rehúyen -por ejemplo- a acercamientos pesimistas y apocalípticos sobre la integración de los seres humanos con máquinas. Hayles (1999), por su parte, apunta a que este no es el fin de lo humano, sino más bien el acceso a nuevas configuraciones culturales de este, diferentes por completo al sujeto del humanismo liberal y su deseo de controlar y dominar, ya que el ser humano interactúa no solo con las máquinas, sino también con su entorno, todo parte de un sistema cognitivo común. Braidotti (2015) -por otro lado-advierte sobre las implicaciones biológicas de 
este proceso de comunión de carne y máquina, a la vez que apuesta a que sea un terreno fértil para pensar en identidades basadas en la transversalidad, es decir, que surjan a través del establecimiento de relaciones con los otros (cibernéticos u orgánicos), así como con el entorno:

Yo creo que el fin de la posición posthumana es reconsiderar la evolución de manera no determinista, y al mismo tiempo, posantropocéntrica. En oposición a las ideas clásicas, lineales y teleológicas de la evolución (Theilhard de Chardin, 1959), me complace evidenciar el proyecto colectivo de encontrar una más aproximada interpretación de la complejidad de factores que estructuran el sujeto posthumano: la nueva proximidad a los animales, a la dimensión planetaria y a los altos niveles de mediación tecnológica (Braidotti, 2015, p. 95).

Parte del movimiento poshumanista, pero con marcadas diferencias a los enfoques de Hayles y Braidotti, la propuesta del Ilamado transhumanista impulsada por Robert Peperell, Hans Moravec y Marvin Minsky, defiende -según Chavarría Alfaro (2015) - "la intervención de la naturaleza humana para llegar más allá de sus límites biológicos" ( $p$. 7). Es decir, este grupo de acaudalados científicos está a favor de realizar una "ciborización" del ser humano, mediante modificaciones genéticas, con el fin de llevarlo a un próximo nivel: incrementar las potencialidades del cuerpo, librarse del envejecimiento, de enfermedades e, incluso, de la muerte.

Obviamente, esta propuesta ha recibido críticas inclusive por parte de los mismos teóricos poshumanistas. Braidotti (2015), una de estas voces críticas, explica que el movimiento transhumanista es una muestra, pero con distinto nombre, de la arrogancia humana. El transhumanismo oculta -según esta autora- el deseo de transcendencia propio de un sujeto individualista y egoísta.

Sin embargo, a pesar de los sueños poshumanos, hay voces divergentes, entre ellas la del ya fallecido David Franklin Noble. Calificado por alguno de sus detractores como tecnófobo, crítico no solo del transhumanismo, sino de cómo se encamina el de- sarrollo tecnológico en la actualidad, Noble -historiador de profesión- es completamente escéptico de las promesas de felicidad, bienestar y progreso, tanto colectivo como individual, derivadas de la alianza entre ciencia y tecnología. Según Sádaba (2009), Noble advierte sobre una sociedad corta de miras, obsesionada con los avances en el campo tecnológico y dócil ante los cantos de sirenas en este campo, sin darse cuenta del peligro latente de una sociedad deshumanizada, en la que las máquinas se convierten en fetiches a idolatrar.

Como último punto controversial en torno al poshumanismo y por ende al cíborg, no podía ser obviado el cometario sagaz de Mejía (2005). Este advierte que gran parte de estas teorías poshumanas provienen de científicos e intelectuales de regiones hegemónicas, quienes proclaman el ocaso del cuerpo, en tanto "(...) los hombres y mujeres occidentalizados de países desfavorecidos apenas reivindican el derecho a comenzar a poseer sus propios cuerpos" (Mejía, 2005, p. 146).

Más allá de las divisiones de tecnófobos y tecnófilos, ¿qué tan lejos se está de los escenarios ya planteados? ¿Son propios de la ciencia ficción y de representaciones culturales ficcionales concretas o más bien pertenecen al día a día? Para Gray (2011), los cíborgs son una realidad muy actual no solo en el campo militar-su principal área de interés-, sino también en lo cotidiano. Es más, la transformación del ser humano a través de la tecnología no es para nada un proceso nuevo: el uso de la tecnología le ha permitido adaptarse, apropiarse del medio y por ende, sobrevivir a través del tiempo. Por lo tanto, se constituye en una forma de evolución artificial, más acelerada que la biológica. El cíborg es, por consiguiente, una escala más en esta evolución. Para Gray (2011), la pregunta a plantearse no es si los cuerpos humanos son cíborgs o no, sino más bien de qué tipo.

De hecho, el mismo Gray (en Córdoba Guardado, 2007) propone tres categorías de cíborgs, de acuerdo con el nivel de integración social humano-máquina:

(...). 1. Simple controllers interfaces informacionales que incluyen redes de 
ordenadores, comunicaciones humano ordenador, vacunas y manipulaciones de informacióngenética;2. Bio-tech integrators, ampliaciones mecánicas simples, como las prótesis médicas, vehículos o sistemas de armamento hombre-máquina; 3. Genetic cyborgs, conexiones hombre-máquina directas como los exoesqueletos militares de vanguardia, así como los planes de descargar conciencias humanas en ordenadores forman parte de esta última categoría (movimiento transhumanista) (p. 261).

Con base en la propuesta de Gray, Sádaba (2009) establece entonces tres tipos de cíborgs, según la integración de lo orgánico con lo inorgánico. El primer tipo consiste entonces en una integración endógena, en la que el ser humano muta con el fin de asimilarse a una máquina. El cuerpo orgánico se modifica (ya sea a través de prótesis, una mecanización, una electrificación) con el fin de potenciarlo. La carne anhela al cero.

En el segundo tipo de integración, en cambio, es la máquina la que más bien imita a lo orgánico. En estos casos, el ser humano -como si fuera un tipo de deidad- crea seres metálicos a su imagen y semejanza. "Nos recuerda, en este caso, al viejo tópico de la inteligencia artificial (IA), a la fabricación de robots pensantes y sensibles, de maquinaria cuasi humana, de réplicas perfectas, indistinguibles del original" (Sádaba, 2009, p. 39).

Finalmente, según Sádaba (2009), en el tercer tipo de cíborg, la costura que divide la carne de lo inorgánico se vuelve más fina, más difusa, incluso imperceptible, pues la fusión entre uno y lo otro surge desde su mismo origen: seres quiméricos, fruto de los desarrollos de la biotecnología y la tecnología, "fusión e hibridación desde la gestación" (p. 39). Se está - entonces- ante el mundo de la manipulación genética.

\section{EL CÍBORG EN CUATRO CUENTOS DE CIENCIA FICCIÓN COSTARRICENSE}

Sentada de manera concisa la discusión en torno a los cíborgs y sus implicaciones, en seguida se ana- liza la selección de textos escogidos, en los cuales es posible hallar su presencia. Antes, eso sí, algunas aclaraciones sobre el corpus escogido. Estos textos han sido todos escritos por distintos autores costarricenses y publicados en dos antologías de ciencia ficción de las primeras dos décadas del siglo XXI: Lunas en vez de sombras y otros relatos de ciencia ficción (2013) y Objeto no identificado y otros cuentos de ciencia ficción (2011).

En un juego intertextual basado en Frankenstein, el clásico de Mary Shelly, José Ricardo Chaves (2013) presenta su versión del cíborg en el texto "Yo, la criatura". A través de un narrador en primera persona, en este cuento se lee la historia de Victoria, cuyo cuerpo -conformado por la unión de seis cadáveres distintos, uno de mujer y el resto de varones- cobra vida gracias a los conocimientos científicos sobre la electricidad que posee el doctor Víctor Frankestein.

Victoria vive -pues- gracias a la simbiosis de componentes orgánicos y un sistema eléctrico. Por ser incombustible, a la que las llamas le producen solo "un cálido aliento sobre la piel" (Chaves et al., 2013, p. 8), Victoria se denomina a sí misma la hija eléctrica del fuego.

Sin embargo, el cuerpo de Victoria presenta otros rasgos todavía más interesantes que la acercan a la noción utópica de cíborg propuesta por Haraway (1984), ya que con su existencia transgrede diversas dicotomías del pensamiento moderno occidental. Para empezar, la genérica: hombre-mujer, masculino-femenino. Si bien su nombre, en clara a alusión al de su padre, es femenino, Victoria no coincide precisamente con ninguna de las clasificaciones genéricas, tampoco con el sujeto cartesiano estable y único. Su identidad es compleja, múltiple, escindida y abyecta, además de gobernada por el lado femenino: “(...) Estoy segura de no ser hombre, aunque dudo de ser mujer. Soy algo que antes no había existido. Los yoes masculinos coexisten conmigo pero yo arreo el rebaño... ¡ Lo que es la fuerza del útero (y del corazón)!..." (Chaves et al., 2013, p. 5).

De igual forma, la constitución y caracterización del personaje también pone en jaque la división entre cultura y naturaleza, lo civilizado y lo primitivo: a pesar de su apariencia monstruosa y animalizada, 
su piel amarillosa, su cuerpo deforme, sus labios negruzcos, su "fuerza y agilidad extraordinarias" (p. 6) y su capacidad de sobrevivir con poca comida, Victoria también posee un gran bagaje cultural (ha leído a Milton, Volney, Plutarco y Goethe). Además, tiene una sensibilidad particular para la música: toca la flauta y canta preciosamente. Amalgama, pues, cualidades notables relativas a la cultura y la naturaleza.

Este carácter híbrido y monstruoso pareciera condenar a Victoria a la soledad. Su creador, Víctor, se niega a engendrar descendencia con ella, a pesar de las constantes intimidaciones y amenazas de Victoria. Ella -ante esta actitud- mata uno a uno a todos los miembros de la familia de Víctor, en una especie de ley de Talión: soledad como castigo de la soledad engendrada.

No obstante, la criatura logra su cometido, ya que copula y concibe un hijo con Ernesto, el hermano de Víctor, cuando este se halla ebrio. La consecución de este embarazo resalta el carácter triunfante, victorioso, del personaje, quien no solo reescribe con su relato la historia oficial, sino que también se acepta y se afirma en su gozosa monstruosidad. De ahí que el título del relato sea un de acto proclamativo de la asunción de su identidad: "Yo, la criatura". Título que -por cierto- es a su vez un guiño intertextual a la colección de relatos Yo, robot del escritor y bioquímico Isacc Asimov.

Publicado también en el mismo cuentario que el texto de Chaves, en "Joyería mental", de Jessica Clark, se está ante la presencia de un cíborg de integración endógena, según la propuesta de Sádaba (2009). La tecnología, específicamente los neuroprogramas creados y comercializados por la doctora Abha, les permiten a sus usuarios acceder a distintas emociones o comportamientos: pánico, amor incondicional, la sensación de fumar opio. En esta lujosa tienda, cada cliente escoge qué emoción "colocarse", según los deseos o necesidades, tal y como si fueran uno de los bindis utilizados por Abha. En este texto de Clark, la irracionalidad de las emociones humanas se somete, con el fin de manipularlas al gusto de cada quien, a través del conocimiento biotecnológico.
Ahora bien, el texto de Clark apunta, desde la lectura de quien escribe, a una insuficiencia de dicho conocimiento: si bien esta tecnología permite modificar la estructura del cerebro y por ende, la producción de ciertas hormonas, su mera existencia no resuelve por completo las crisis existenciales del ser humano. Este fracaso se evidencia en el texto con la historia de Jake, hombre inteligente, con apariencia de "rebelde adinerado" e íntimo amigo de la doctora Abha.

Jake guarda un oscuro secreto en su pasado, no aclarado por completo en el texto: de alguna manera, el personaje estuvo involucrado en el asesinato de su amigo Joao. El peso de esta culpa provoca que Jake consuma constantemente alcohol, sustancia que lo hace sentir momentáneamente mejor (un placebo menos sofisticado que la joyería mental). Con la intención de ayudarlo, Abha crea los neuroprogramadores y lleva 4 años pensando en el ideal para Jake, con la idea de ofrecérselo como regalo. Donado por un astronauta, el original obsequio consiste en "la reacción de la mente al sonido del sol" (Chaves et al., 2013, p. 19).

A pesar de su originalidad, este maravilloso regalo no alivia la carga y la culpa de Jake. Paradójicamente, las palabras de consuelo de su amiga resultan ser más efectivas. Por eso ante la pregunta de si las pastillas lo ayudarán a olvidar lo sucedido, Abha le responde que no, pero le ofrece como consuelo las siguientes palabras: "—De por sí, Joao no era buena persona-murmuró ella" (p. 20). La reacción de su amigo evidencia el poder de estas: "Jake sonrió en la penumbra" (Chaves et al., 2013, p. 20).

Escrito en primera persona, en "Lunas en vez de sombras" (2013), de Ericka Lippi, la configuración del cíborg en este texto opera de una manera similar al previamente analizado de Clarke, ya que se está de nuevo ante un cíborg perteneciente a la segunda categoría según Grey. Esta vez el objeto que provoca la modificación de la parte biológica es una pastilla llamada "Psoria", medicamento que mejora la productividad de aquel que la consume:

Se trata de una oportunidad para que, tanto el hemisferio izquierdo como el derecho, logren un equilibrio. Si quiere un cambio y 
desempeñarse con excelencia en todos los aspectos de su vida, consuma Psoria. Maximizará sus niveles de energía. La energía negativa se convertirá en energía positiva. Sea mejor padre o madre de familia, mejor hijo o hija o mejor estudiante (Chaves et al., 2013, p. 49).

El "Psoria" se convierte en un medicamento idóneo para la sociedad descrita en el texto, obsesionada no solo por el rendimiento eficaz y eficiente de los cuerpos ("maximizar los niveles de energía", como se indica en la cita anterior), sino también por moldearlos según un estándar de belleza y salud, particularmente importantes en una sociedad agobiada por una epidemia de obesidad. Sue, la vOz narradora y personaje principal del texto, es parte de esta industria tecnológica: como nutricionista, ella trabajó en la compañía encargada de diseñar y fabricar "Psoria".

Sin embargo, a pesar de todas las ventajas que aparenta producir, "Psoria" presenta un serio efecto secundario: provoca grandes lagunas mentales en su consumidor, quien será incapaz de recordar momentos cercanos en su pasado. Sue -por ejemplo-no logra recordar qué hizo ni con quién ha estado durante las noches. Ella entra en un "trance de luna", de ahí la constante referencia en el texto a la canción de la violinista Lindsey Stirling que Ileva justamente el nombre de Moon Trance.

El tiempo, pues, pasa a ser uno de los principales puntos de reflexión del relato: ¿qué significa utilizar el tiempo eficientemente?, ¿cumplir a cabalidad con un horario de labores?, ¿vale la pena vivir sin poder recordar?, ¿qué es el ser humano sin su capacidad de memoria? No en vano, Sue piensa constantemente en La persistencia de la memoria, el famoso cuadro de Dalí, para simbolizar estas interrogantes:

Tengo la sensación de que la noche transcurrió en tres segundos. Los relojes blandos de Salvador Dalí vienen a mi mente. Me miro la mano. Algunas veces tengo la impresión de que mis manos se encuentran mojadas por el tiempo que se escurre en mis manos (Chaves et al., 2013, p. 46).
Como se observa, si bien la pastilla permite mejorar la capacidad de producción y concentración de quien la toma, para convertirse en un eficiente y productivo cíborg, este proceso no resuelve las eternas reflexiones sobre la inexorabilidad del tiempo y la fugacidad de la vida. En este sentido, al igual que en el texto anterior ("Joyería mental"), el proceso de convertirse en un cíborg no hace más sencillos los dilemas existenciales o éticos de los personajes.

De hecho, además de la reflexión del tiempo ya aludida, Sue también se enfrenta a un cuestionamiento respecto a su ética profesional, al descubrir que el anodino y cortante nuevo cliente es en realidad un sofisticado androide, creado como parte del nuevo proyecto secreto de su exjefe y amante Mike, hombre brillante, pero ambicioso y capaz de transgredir las reglas o engañar para hacer dinero:

Sabía que era un hombre sumamente capaz como químico y físico, con una facilidad excepcional para el mercadeo y una capacidad de convencimiento tan extraordinaria que rozaba la hipnosis. También conocía cómo había cedido a ciertas presiones por una ambición insana (Chaves et al., 2013, p. 48).

Al final del relato, Sue debe decidir qué hacer: colaborar con el proyecto de Mike, con todas sus implicaciones éticas y concretar su estabilidad económica o-por el contrario- cortar de raíz, alejarse tanto de Mike como de sus intenciones de crear androides, aunque esto implique un futuro económico incierto. La respuesta a esta incógnita queda abierta al cierre del texto.

Con un abordaje diferente a la figura del cíborg, en el último texto por analizar, "El ejército de Onara", de Daniel Garro, se narra la historia de la almirante Liz Benet, asignada junto con su ejército (la Compañía de $C$ de Impacto Terrestre) a Onara, con la finalidad inicial de destruir el laboratorio de Coli, un niño genio, "tal vez de ochos años en la medida terrestre" (p. 55), quien en sus experimentos creó seres híbridos, monstruos; en palabras de Benet: "mezclas y más mezclas horribles de personas y animales" (Castillo et al., 2011, p. 43). 
El laboratorio de Coli es destruido con todos y cada uno de sus experimentos, de sus hijos, como él los Ilama. Un año después, el científico se venga y destruye todo el ejército de Benet, excepto a ella, como un acto de retribución por haberle perdonado la vida en la destrucción de su laboratorio.

Como se observa, según la trama del texto, el cuestionamiento sobre los límites de la experimentación con humanos es clave para interpretarlo no solo por los experimentos realizados por Coli, sino también por la misma conformación del ejército liderado por Benet, compuesto por cíborgs militarizados:

(...) los soldados Genélite, de alto desempeño, altos, fornidos, un poco brutos, de brazos y piernas tan musculosos y arqueados que más bien lucían redondos, con cañones y armas en los antebrazos, un pesado casco electrónico, y los miembros y el torso cubiertos de prótesis de defensa y maximización física (...) (Castillo et al., 2011, p. 34).

Estas modificaciones les permiten a estos soldados tener más fuerza y resistencia física, pero también los convierte en seres grotescos y animalizados: antes del enfrentamiento, gruñen, escupen y se frotan los dientes como rottweilers. En este caso, la tecnología bélica potencia la parte biológica del cuerpo humano de los soldados, armas sofisticadas a la misma vez que seres degradados, irracionales, impulsivos y rabiosos. De hecho, estos rasgos terminan provocando la muerte de los Genélite; uno de ellos -Torre- se descontrola pues cree que van a ser atacados. En realidad, la paranoia del soldado se activa ante el juego de luces y sonidos que Coli les presenta a manera de trampa. Al creerse atacado, el soldado abre fuego indiscriminadamente, lo cual inicia una confusión entre el resto de sus compañeros, quienes se disparan entre sí y terminan matándose.

Esta monstruosidad de los cuerpos cíborgs de los soldados contrasta con la belleza de Benet: “(...) mujer imponente, alta, robusta y hermosa a sus cincuenta años, con un físico poderoso y esbelto que hacía preguntarse: ¿el uniforme la hace lucir bien, o tal vez ella hace lucir bien al uniforme?" (p. 40). Este personaje tampoco ha sufrido modificación alguna y encarna, en contraste con la mayor parte de sus subordinados, características como la misericordia, la compasión, incluso actúa en ocasiones de forma maternal. Para Coli, ella resulta un interesante contraste en comparación con la brutalidad de los soldados modificados genéticamente; de ahí que con curiosidad le diga: “¡Eres tan diferente!" (Castillo et al., 2011, p. 56).

El origen de estos soldados cíborgs es también otro de los puntos llamativos del texto. Según se supone por las palabras de Brolin -uno de los tantos Genélite- él se convirtió en un cíborg luego de ser herido brutalmente en la cabeza en un enfrentamiento militar: "-Mmmme hirieron en Arinax 3-dijo Brolin, con un aire de orgullo que logró colarse entre sus muecas de histeria -P-p-prácticamente....me volaron la cabeza, ipe-pero acá estoy!" (Castillo et al., 2011, p. 39).

Según la cita anterior, se deduce que se experimentó con soldados en condición crítica. ¿Quién decidió entonces que ellos serían intervenidos? ¿Son estos soldados producto también de "jueguitos" y experimentaciones genéticas similares a las de Coli? ¿Cuál es pues la diferencia entre un bando y otro? ¿Si Coli es un monstruo al experimentar y crear seres híbridos, pertenecientes al tercer tipo de integración, según Sádaba (2009), qué diferencia hay entonces entre el niño científico y aquellos que ordenaron la creación de los Genélite?

No deja también de ser llamativa la forma en que se resuelve el texto: al final, los únicos sobrevivientes son aquellos personajes humanos en su totalidad: Coli y Benet. Ambos vivos gracias a que, en algún momento de la trama, fueron objeto de la misericordia de otra persona.

A grandes rasgos, en este texto el cíborg no se vincula con la trascendencia del ser humano a un plano superior, sino con su degradación: en manos equivocadas, como indica Sádaba (2009) en un parafraseo de Goya, el sueño de la razón (de la biotecnología, en este caso) produce monstruos. La pregunta clave reside entonces en cuestionarse quiénes son los verdaderos monstruos: aquellos seres que 
fueron modificados para fines bélicos o quienes los crearon.

\section{CONCLUSIONES}

Para cerrar este análisis, se destaca que en la mayor parte de los textos analizados el cíborg alerta sobre los peligros de la tecnología y sus límites éticos (tal y como ocurre, por ejemplo, en "El ejército de Ona$\left.\mathrm{ra}^{\prime \prime}\right)$. En este caso, la incorporación de la tecnología animaliza degrada al que la utiliza y vuelve monstruoso tanto al usuario como al creador.

En tanto, en "Joyería mental" y en "Lunas en vez de sombras", la tecnología resulta incapaz de resolver las preocupaciones inherentes a la existencia humana. Llama la atención también que, en estos casos, la gradación del cíborg no abarca el nivel 3 -según la escala de Grey-, lo cual podría implicar que la humanidad en los personajes no se pone entredicho, a diferencia del texto de Garro. El proceso de convertirse en un cíborg se relaciona más bien en ambos textos con un uso hedonista; la tecnología y las posibilidades que esta brinda para convertirse en cíborg se presentan como un bien de consumo anhelado.

Por su parte, en "Yo la criatura" la subjetividad de Victoria reta por completo las dualidades básicas del pensamiento occidental. Esta transgresión al modelo de humano es un proceso gozoso para Victoria, orgullosa de su monstruosidad. En este caso, el cíborg sale bien librado, ya que consigue sus objetivos (no estar solo, formar una familia).

Como palabras finales, como bien indica Sádaba (2009), ante el eminente auge de los descubrimientos en el área científica y tecnológica, la literatura de ciencia ficción se encarga de presentar al lector esos "futuros imaginados" (p. 31). Estos textos funcionan entonces como un espacio catártico (y por ende, tranquilizador) sobre los miedos en torno a un presente dominado por el conocimiento tecnocientífico, pero además "articulan una auténtica economía política de la esperanza" (p. 231), pues las profecías anunciadas en ellos todavía no llegan, es decir, hay aún tiempo para cambiar el futuro. Además, como advierten Gray (2011) y Chavarría Alfaro (2015), el desarrollo de estas nuevas tecno- logías debe formar parte de una discusión pública y democrática, porque se corre el riesgo de que el debate se haga a puertas cerradas, con la participación de algunos pocos privilegiados. La ciencia ficción (y las reflexiones y preocupaciones inherentes a ella) resulta entonces una valiosa antesala a este diálogo tan necesario.

\section{REFERENCIAS BIBLIOGRÁFICAS}

Alfaro Vargas, R. (2014). El Novum en la ciencia ficción costarricense. Revista de Filología y Lingüística de la Universidad de Costa Rica, 40 (1) 129-140. Recuperado de http://revistas.ucr. ac.cr/index.php/filyling.

Braidotti, R. (2015). Lo posthumano. Recuperado de http://ebookcentral.proquest.com.

Brown, A. (2010). Cyborgs in Latin America. Nueva York, Estados Unidos: Palgrave Macmillan.

Castillo, M., Clark, J., Delgado, M., Ortiz, A., Quijano, L., Ugalde, E. (2011). Objetos no identificados y otros cuentos de ciencia ficción. San José, Costa Rica: EUNED.

Chavarría Alfaro, G. (2015). Poshumanismo y los cambios en la identidad humana. Revista Reflexiones, 94 (1) 97-107. Recuperado de http:// revistas.ucr.ac.cr/index.php/reflexiones/article/ view/20882.

Chaves, J., Clark, J., González Chaves, D., Lippi Rojas, E., Molina Jiménez, I., Rossi, A. (2013). Lunas en vez de sombras y otros relatos de ciencia ficción. San José, Costa Rica: EUNED.

Córdoba Guardado, S. (2007). La representación del cuerpo futuro [Tesis para optar al grado de Doctor en Bellas Artes]. Universidad Complutense; Madrid, España. Recuperado de http:// eprints.ucm.es/7536/.

Cuvardic García, D. (2014). Capitalismo voraz y cuerpos consumidos: distopía postnacional y globalización en Fragmentos de la tierra prometida, de Fernando Contreras. Revista de Filología y Lingüística de la Universidad de Costa Rica, 
40 (1) 115-126. Recuperado de http://revistas. ucr.ac.cr/index.php/filyling.

Eco, U. (1998). De los espejos y otros ensayos. Barcelona: Editorial Lumen.

Gray, C. H. (2011). Homocyborg: cincuenta años después. Teknocultura. Revista de Cultura Digital y Movimientos Sociales, 8 (2) 83-104.

Gray, C. (1999). Human Potencial, the Information Society and Ciborgiation. Recuperado de http:// www.econstor.eu/bitstream/10419/114261/1/ ERSA1999_a124.pdf.

Gunn, J. (2005). Speculations on Speculations: Theories of Science Fiction. Lanham-TorontoOxford: Scarecrow Press.

Haraway, D. (1984). Manifiesto cíborg. Descargado desde https://xenero.webs.uvigo.es/profesorado/beatriz_suarez/ciborg.pdf.

Hayles, K. (1999). How we became posthuman: virtual bodies in cybernetics, literature, and informatics. Chicago, Estados Unidos: University of Chicago Press.

Malmgren, C. D. (1988). Towards a Definition of Science Fiction. Science Fiction Studies 15 (46): 259-281.

Mejía, I. (2005). El cuerpo poshumano en el arte y la cultura contemporánea. México: UNAM. Recuperado de http://es.scribd.com.

Molina Jiménez, I. (2015). León Fernández Guardia y "El número 13,013". Alambique: Revista académica de ciencia ficción y fantasía, 3 (1) 1-15. Recuperado de http://scholarcommons.usf.edu/ alambique/vol3/iss1/1/.

Molina Jiménez, I. (enero, 2010). Género en construcción: la ciencia ficción costarricense. A propósito de Vuelo de Ra, de Manuel Delgado. Acontracorriente. Revista de Historia y Literatura en América Latina, 8 (1) 408-415. Recuperado de http://www.ncsu.edu/project/acontracorriente.

Moreno, F. (2010). Teoría de la Literatura de Ciencia ficción. Poética y retórica de lo prospecti- vo. Vitoria, España: Portal Editions. Recuperado de https://www.academia.edu/11817054/ Teor\%C3\%ADa_de_la_Literatura_de_cf_primera_edici\%C3\%B3n_

Pacheco Solórzano, C. (enero-junio, 2014). Discursos literarios en Costa Rica. Revista Espiga, 13 (27), 59-66. Recuperado de investiga.uned. ac.cr/revistas/index.php/espiga.

Ruiz Ulloa, G. (2014). Entredichos de la sociedad democrática costarricense: Ciencia ficción y política. Revista de Filología y Lingüística de la Universidad de Costa Rica, 40 (1) 107-112. Recuperado de http://revistas.ucr.ac.cr/index.php/ filyling.

Sádaba, I. (2009). Cyborg. Sueños y pesadillas de las tecnologías. Barcelona, España: Ediciones Península.

Soto Bogantes, C. (2014). El mundo natural y el futuro de la colonialidad: "Frente frío" de Jessica Clark y "La flor del crepúsculo" de Laura Quijano. Revista de Filología y Lingüística de la Universidad de Costa Rica, 40 (1) 87-95. Recuperado de http://revistas.ucr.ac.cr/index.php/filyling.

Víquez Jiménez, A. (2014). Lectura de dos relatos de ciencia ficción costarricense "Órdago" y "Objeto no identificado". Revista de Filología y Lingüística de la Universidad de Costa Rica, 40 (1) 87-95. Recuperado de http://revistas.ucr. ac.cr/index.php/filyling. 